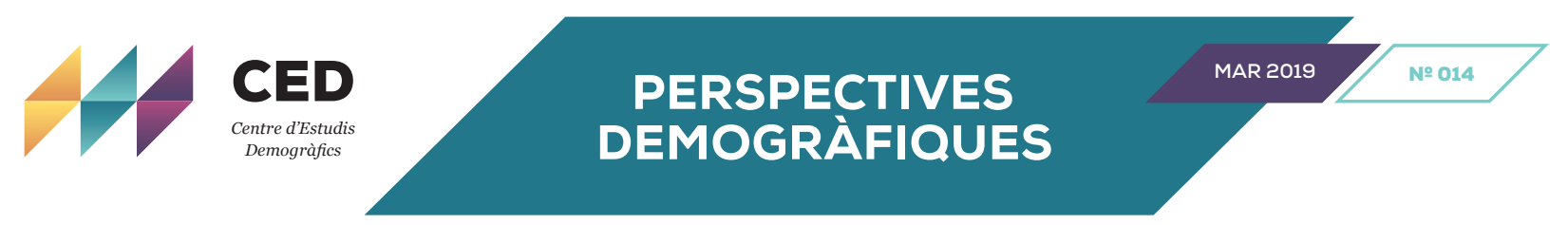

\title{
La persistente desigualdad de género en el uso del tiempo en España
}

\author{
Marc Ajenjo y Joan García Román, Centre d’Estudis Demogràfics
}

Las relaciones de género son un elemento esencial para entender las dinámicas de la formación y disolución de las parejas y sus niveles de fecundidad en el mundo occidental. Argumentos teóricos recientes postulan que la transición hacia una mayor simetría de género debería de estimular la formación de parejas y la fecundidad. Un ejemplo de ello es la recuperación de la fecundidad que ha tenido lugar en los países escandinavos. En este contexto, el aumento continuo del nivel educativo y la ocupación de la población femenina ayudaría a activar esa transición. A pesar de ello, diversos estudios apuntan que las desigualdades de género en la distribución del tiempo persisten y tienden a aumentar con la llegada de hijos. En este número de Perspectives Demogràfiques examinamos con detalle las diferencias de género en el uso del tiempo en España, partiendo de la situación familiar de las personas y, en el caso de les parejas, de la posición relativa de la mujer respeto al hombre en relación al nivel de estudios y contribución a los ingresos del hogar.

Con datos de la Encuesta de Empleo del Tiempo 2009-2010 (INE), analizamos el tiempo diario que unos y otras dedican a las actividades cotidianas en distintos momentos del ciclo de vida. Entre las principales conclusiones hay que destacar que la desigualdad ya se produce en la infancia, pero que es en la edad adulta y con el nacimiento de la descendencia, cuando más incrementa. Las desigualdades de género se mantienen, aunque no en la misma magnitud, incluso entre las parejas en las que ellas tienen un mayor nivel educativo y unos ingresos superiores.

\section{LA PERSISTENTE DESIGUALDAD A LO LARGO DEL CICLO DE VIDA}

Aunque en las últimas décadas en la mayoría de sociedades occidentales se ha producido una cierta convergencia en la distribución de los usos del tiempo de hombres y mujeres, la brecha de género continúa siendo considerable (Kan et al 2011). La plena incorporación de la mujer al trabajo remunerado no ha tenido como contrapartida la del hombre al trabajo no remunerado, al cual solo recientemente se ha dedicado en una parte, la que se refiere el cuidado de los niños. Así lo muestran algunos artículos que analizan esta cuestión en otros países occidentales, concluyendo que, con altibajos, las diferencias en el reparto del tiempo se observan a lo largo de todas las etapas del ciclo de vida (Anxo et al., 2011). En esta línea, el primer objetivo que nos proponemos aquí es dimensionar estas diferencias en la España actual.

A partir de los datos de la Encuesta de empleo del tiempo llevada a cabo por el INE en 2009-2010, nos hemos aproximado a los usos del tiempo en distintos momentos del ciclo de vida. Para ello, en función de FIGURA 1. Media de horas dedicadas a trabajo remunerado, trabajo doméstico,
cuidado de menores y ocio en día laborable. Hombres y mujeres en distintas etapas
del ciclo de vida

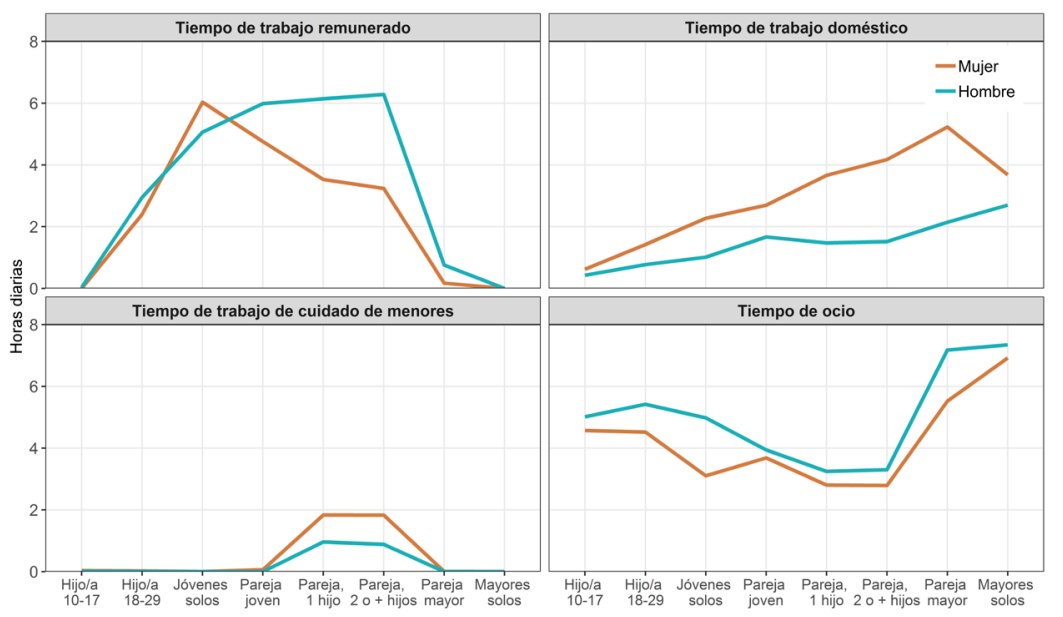

Fuente: Elaboración propia a partir de los datos de la Encuesta de Empleo del Tiempo (INE, 2009-2010) 
su edad y situación familiar, asumimos que los individuos se encuentran en un determinado momento del ciclo de vida, estimando, para este momento, el tiempo dedicado en un día laborable a cuatro actividades: trabajo remunerado, trabajo doméstico, trabajo de cuidado y ocio.

Evidentemente las diferencias en las biografías personales comportan que las etapas por las cuales transitan los individuos a lo largo de su vida no sean las mismas, ni se cursen en el mismo orden. Para simplificar, hemos considerado ocho etapas que no abarcan, ni mucho menos, todas las situaciones: dos en el rol de hijos/as que conviven con sus progenitores (a las edades 10-17 y 18-29 años), otra etapa de joven viviendo solo (menores de 35 años), cuatro más de convivencia en pareja (pareja joven sin hijos, pareja con un hijo, pareja con dos hijos, pareja mayor sin hijos), mientras que la última la constituiría las personas mayores que viven solas.

La distribución del tiempo que hombres y mujeres emplean en las cuatro actividades a lo largo de las ocho etapas mencionadas se pueden leer en la figura 1, dónde se observan diferencias importantes en las cuatro actividades y en todas las etapas del ciclo de vida: los hombres dedican más tiempo a trabajo remunerado y ocio y las mujeres a trabajo doméstico y a cuidado de menores. Pero veámoslo con más detalle.

La diferencia entre hombres y mujeres se inicia, como mínimo, en la adolescencia, cuando niños y niñas viven con sus progenitores. A pesar de que en esta etapa el tiempo dedicado al trabajo doméstico es poco importante, las niñas destinan a estas tareas una media hora diaria más que sus hermanos varones. Las diferencias entre ellos y ellas aumentan ligeramente con la edad, de modo que ascienden hasta los 45 minutos diarios entre los 18 y 29 años, lo que se traduce en un mayor tiempo de ocio por parte de ellos.

Cuando se emancipan -jóvenes solos-, los hombres siguen con una menor dedicación a las tareas domésticas que las mujeres. Y si añadimos que es el único momento del ciclo de vida en que las mujeres dedican más tiempo al trabajo remunerado que los hombres, la consecuencia es un incremento considerable en la diferencia en el tiempo de ocio, que llega hasta las dos horas a favor de ellos. Teniendo en cuenta que se trata de personas jóvenes que viven solas, la diferencia observada en el tiempo empleado en trabajo doméstico tiene dos posibles explicaciones: existe una forma distinta de realizar las tareas domésticas -ya sea recurriendo más a comida preparada y/o adoptando un menor estándar de limpieza, por ejemplo-, y/o existen importantes diferencias en cuanto a la ayuda externa -de pago o familiar- en algunas de estas tareas.
Las diferencias se mantienen cuando hombres y mujeres empiezan a convivir, de modo que, en las parejas jóvenes sin hijos, ellas dedican una hora diaria más a tareas domésticas que sus homólogos. Esta diferencia puede explicarse en parte -pero solo en parte- por una mayor participación del hombre en el mercado laboral: al analizar esta diferencia solo para la población ocupada, aunque esta hora se reduce, ni mucho menos desaparece.

Ahora bien, el principal detonante de la desigualdad en los usos del tiempo es el nacimiento de hijos e hijas. Aunque es verdad que una parte del incremento observado en las diferencias es una cuestión generacional, diversos estudios han confirmado que la mayor parte no se debe a un cambio generacional y que, a pesar de que las nuevas cohortes tienen unos ideales más igualitarios, se produce cierta tradicionalización de comportamiento con la llegada de la descendencia (Abril et al., 2015; Ajenjo y García-Román, 2011). El nacimiento de los hijos desencadena, en las mujeres, una caída de la actividad remunerada y un aumento tanto de tareas domésticas como de cuidado. A grandes rasgos, observamos que las mujeres con hijos destinan al conjunto de tareas domésticas y de cuidado, 4,5 horas más que sus parejas. Si se considera el tiempo como un juego de sumas y restas y se define el tiempo de trabajo total como la suma de trabajo remunerado, doméstico y de cuidado a lo largo de la semana -incluyendo sábados y domingos-, concluimos que en las parejas que tienen hijos, las mujeres dedican a este trabajo 5,5 horas más que sus prójimos.

En cierta medida, la brecha en dedicación al trabajo doméstico se mantiene en la etapa que habitualmente se conoce como nido vacío. Y se mantiene a pesar del fuerte descenso en las diferencias observadas en el trabajo remunerado, circunstancia que comporta una importante desigualdad en ocio. Por otro lado, y a pesar de que se trata de generaciones muy distintas, las personas mayores que viven solas siguen un patrón similar - sin trabajo remunerado- al de las personas jóvenes que viven solas.

\section{LA PERSISTENTE DESIGUALDAD EN LAS PAREJAS CON HIJOS}

Así pues, la llegada de descendencia comporta un importante incremento en las diferencias de género en el uso del tiempo. Pero, ¿hasta qué punto las características de la pareja pueden atenuar este incremento? Y, ¿qué sucede cuando es la mujer quién tiene una mejor posición en el mercado de trabajo, en el nivel de estudios o tiene un nivel de ingresos superior al de su pareja? Para responder a estas preguntes nos hemos centrado en parejas con hijos menores donde la mujer tiene entre 20 y 49 años. 
FIGURA 2. Distribución del trabajo doméstico y de cuidado de menores por parte de hombres y mujeres, en función de la relación con la actividad de la pareja. Parejas con hijos en las que la mujer tiene 20-49 años
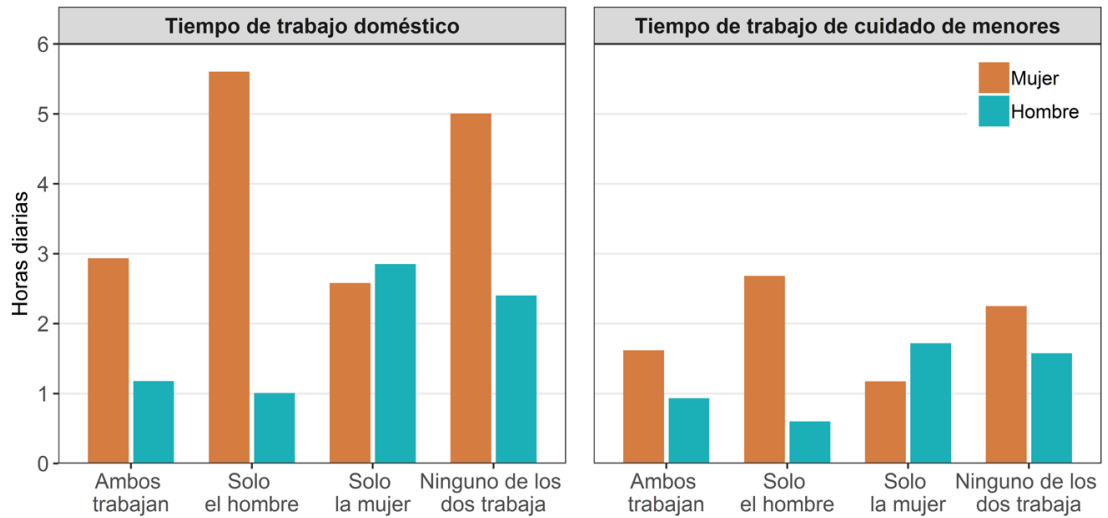

Fuente: Elaboración propia a partir de los datos de la Encuesta de Empleo del Tiempo (INE, 20092010).

La figura 2 muestra el tiempo dedicado al trabajo doméstico y de cuidado según la relación con la actividad de ambos miembros de la pareja. Excepto en aquellas parejas en las que solo trabaja la mujer, donde los hombres dedican unos quince minutos más al trabajo doméstico y una media hora más al cuidado que las mujeres, las tareas de trabajo no remunerado recaen principalmente en ellas. En concreto, entre las parejas mayoritarias, aquellas en que ambos se encuentran ocupados, la brecha de género en trabajo doméstico se puede cifrar en una hora y cuarenta y cinco minutos, y en unos cuarenta minutos en el caso del cuidado de menores. Huelga decir que, aunque los dos miembros de la pareja se encuentren ocupados, no significa que tengan la misma jornada laboral: los hombres que trabajan dedican una media de dos horas más al mercado de trabajo que sus parejas. Si repetimos para las parejas de doble ingreso con hijos el juego de sumas y restas a lo largo de una semana, obtenemos que el tiempo de trabajo total es unas cinco horas superior en ellas que en ellos.

A pesar de que, en cuanto a su situación laboral, las parejas donde solo trabaja la mujer tienen una configuración simétrica a aquellas en las que solo trabaja el hombre, el tiempo dedicado a trabajo doméstico y de cuidado no se revierte, ni mucho menos. Así como tampoco son equivalentes, sobre todo en tiempo empleado en trabajo doméstico, la situación en la que ambos trabajan y aquella en la que están los dos desocupados. mujer tiene 2O-49 años

\section{LA PERSISTENTE DESIGUALDAD CUANDO LA MUJER TIENE UN CAPITAL HUMANO SUPERIOR AL DE SU PAREJA}

En las últimas décadas se ha producido una mejoría del nivel educativo de la mujer, que ha pasado a ser superior al de los hombres y que ha supuesto la proliferación de parejas en las que ella tiene un mayor nivel educativo que él (Esteve et al. 2012). Según las teorías explicativas de la división de roles en el hogar, la mejor educación de la mujer, que permite una mejor ocupación y nivel de ingresos, debería traducirse en un mayor poder negociador repercutiendo en un reparto más equitativo de tareas. La figura 3 muestra el reparto del trabajo no remunerado en las parejas de doble ingreso según su nivel educativo.

La figura muestra que, si bien cuando ella tiene un nivel educativo superior al de su pareja las disparidades se reducen, la mujer es, en todas las situaciones, la principal responsable del trabajo doméstico y de cuidado (esta lógica también se repite si se representa el nivel de ingresos). La menor divergencia no baja de la hora y 25 minutos, y es muy similar entre las parejas en las que ambos miembros tienen como mínimo el bachillerato y aquellas en las que ella tiene un nivel educativo superior a él.

En el otro extremo, las mayores diferencias se observan en las parejas donde ambos miembros tienen estudios secundarios o inferiores, dos horas y veinte minutos. A pesar de que en estas parejas, el tiempo empleado por parte de las mujeres es similar al de las parejas formadas por un hombre de mayor nivel educativo, se observa una desigualdad importante: en estas últimas, los hombres emplean

FIGURA 3. Distribución del trabajo doméstico y de cuidado de menores por parte de hombres y mujeres, según el nivel educativo de la pareja. Parejas de doble ingreso con hijos en las que la
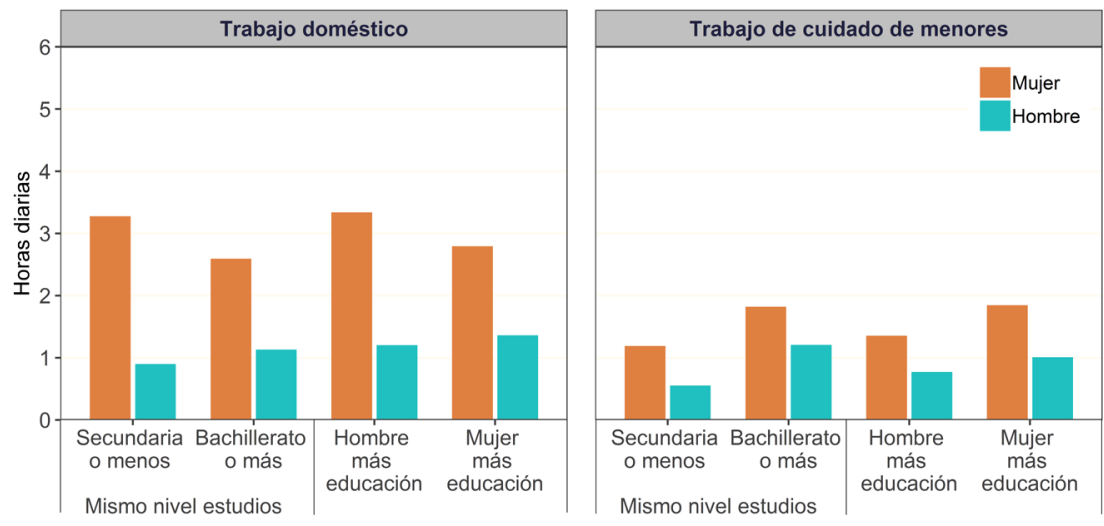

Fuente: Elaboración propia a partir de los datos de la Encuesta de Empleo del Tiempo (INE, 2009-2010). 
unos veinte minutos más al trabajo doméstico y unos quince más al cuidado que en las parejas donde ambos tienen un nivel inferior al bachillerato.

En general, se observa una menor brecha de género en el tiempo de cuidado, un tiempo que viene mayormente condicionado por las características del individuo y no por las características de su pareja. Tal y como muestran estudios recientes (Borràs et al., 2018), el aumento e intensificación de la crianza en los últimos años ha ido acompañado de un importante incremento en el tiempo de cuidado por parte de los hombres sin que ello haya comportado un descenso del tiempo de cuidado de sus parejas.

Pero, ¿̇cómo son las parejas de doble ingreso con hijos menores en las que se da una mayor igualdad? ¿Llegan a alcanzar la igualdad en tiempo doméstico y de cuidado? En cuanto a la primera pregunta, debemos especificar, antes de nada, que las características de la mujer son mucho más importantes que las de su pareja y, en segundo lugar, en relación a sus rasgos esenciales, diremos que se trata de parejas en las que la mujer tiene estudios universitarios y unos ingresos superiores a 2.000€, una condición que solo cumplen aproximadamente el 6\% de las parejas de doble ingreso con hijos.

Ahora bien, y a pesar de la importante reducción de las diferencias, ni en esta situación se consigue la plena igualdad. Tal y como muestra la figura 4, en este colectivo las mujeres siguen dedicando diariamente una media hora más al trabajo doméstico y media hora más al cuidado que sus parejas. La principal característica de este colectivo es que dispone, en gran medida, de servicio doméstico, siendo esta la principal vía para alcanzar un menor nivel de desigualdad. Un servicio doméstico que compensa, en gran parte, el trabajo doméstico que en el resto de parejas realiza la mujer. Y es que, tal y como se observa si comparamos los hombres de este colectivo con los de la figura 3 ,

\section{Referencias bibliográficas}

Abril, Paco et al. (2015). "Ideales igualitarios y planes tradicionales: análisis de parejas primerizas en España”. Revista Española de Investigaciones Sociológicas, 150: 3-22.

Ajenjo, Marc; García-Román, Joan (2011). "El tiempo productivo, reproductivo y de ocio en las parejas de doble ingreso". Papers, 96 (3): 9851006

Anxo, Dominique et al. (2011). "Gender Differences in Time Use over the Life Course in France, Italy, Sweden and the US". Feminist Economics, 17 (3): 159-195.

Borràs, Vicent; Ajenjo, Marc; Moreno-Colom, Sara (2018). "More time parenting in Spain: a possible change towards gender equality?". Journal of Family Studies, DOI: 10.1080/13229400.2018.1440618
Esteve, Albert; García-Román, Joan; Permanyer, Iñaki (2012). “The gender-gap reversal in education and its effect on union formation: the end of hypergamy?". Population and Development Review, 38 (3): 535-546.

Kan, Man Yee; Sullivan, Oriel; Gershuny, Jonathan (2011). "Gender Convergence in Domestic Work: Discerning the Effects of Interactional and Institutional Barriers from Largescale Data”. Sociology, 45: 234-251.

\section{Cita}

Marc Ajenjo y Joan García Román (2019) "La persistente desigualdad de género en el uso del tiempo en España”. Perspectives Demogràfiques, 14: 1-4.

\section{Editores}

Andreu Domingo y Albert Esteve

el tiempo empleado en trabajo doméstico no difiere demasiado entre unos y otros -poco más de una hora-, sino que es el tiempo de la mujer el que se reduce de forma significativa.

FIGURA 4. Distribución del trabajo doméstico y de cuidado de menores por parte de hombres y mujeres. Parejas de doble ingresos con hijos, donde la mujer tiene educación universitaria licenciatura o más) y gana más de $2.000 €$

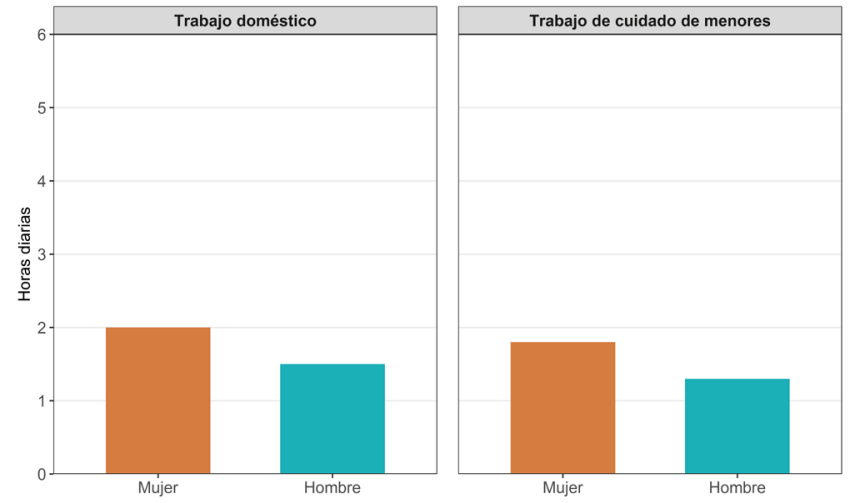

Fuente: Elaboración propia a partir de los datos de la Encuesta de Empleo del Tiempo (INE, 2009-2010)

\section{¿ESTAMOS EN EL CAMINO HACIA UNA MA- YOR IGUALDAD?}

Las mejoras en la igualación de los papeles de género en una sociedad que se pretende moderna, como la española, encuentra uno de sus límites en el micro-poder del hombre que proviene del resultado del empleo del tiempo invertido en tareas reproductivas. Incluso las parejas mejor situadas desde el punto de vista de su capital humano solo consiguen aproximarse a la igualdad externalizando el trabajo doméstico. Una solución que reduce, pero no elimina, las diferencias en el seno de la pareja, pero que, no lo olvidemos, conlleva perpetuar la feminización del trabajo doméstico.

\section{Correspondencia dirigida a}

Marc Ajenjo

majenjo@ced.uab.cat

Joan García Román jgarcia@ced.uab.cat

\section{Créditos}

Gráficos: Anna Turu Maquetación: Xavier Ruiz Vilchez

Enlace url

http://ced.uab.es/es/difusion/ butlleti-perspectives-demografiques

Agradecimientos

A Rocío Treviño por sus comentarios siempre acertados sobre el texto.

Esta investigación ha sido posible gracias al apoyo del programa Beatriu de Pinós de la AGAUR (2016 BPo0279) y al proyecto de investigación (CSO2016-78715-R).

\section{Contacto}

Centre d'Estudis Demogràfics. Calle de Ca n'Altayó, Edificio E2 Universitat Autònoma de Barcelona o8193 Bellaterra / Barcelona España

Teléfono: +34 935813060 E-mail: demog@ced.uab.es Web: http://ced.uab.es/es/ 